\title{
Integrated analysis of microbiota with bile acids for the phototherapy treatment of neonatal jaundice
}

\author{
Kun Zhang, Sainan Fan, Anping Lv, Yanan Ma, Xiaohui Fang, Jinping Zhang
}

Department of Pediatrics, Shanghai Jiao Tong University Affiliated Sixth People's Hospital, Shanghai, China

Submitted: 19 February 2021

Accepted: 6 March 2021

Arch Med Sci

DOI: https://doi.org/10.5114/aoms/134023

Copyright $\odot 2021$ Termedia \& Banach

\section{Abstract}

Introduction: Infant jaundice is a common condition which results from a high concentration of serum bilirubin. Phototherapy is a widely used treatment for bilirubin clearance. We analyzed the effect of phototherapy on intestinal flora and metabolism of newborns. The aim was to assess the benefit of treatment for hyperbilirubinemia with phototherapy.

Material and methods: Fifty-three jaundiced infants hospitalized at our neonatal intensive care unit were treated with phototherapy. Of them, 29 were prescribed antibiotics during the hospitalization. Fecal samples were collected before and $24 \mathrm{~h}$ and $48 \mathrm{~h}$ after phototherapy. The bacterial species and relative abundance were identified with Macrogene sequencing. The bile acids in feces were identified using liquid chromatography-mass spectroscopy (LC-MS).

Results: Differential microbial species/genera and secondary bile acids were found after phototherapy. There are significant differences in the changes of the microbial species/genera between infants who did not receive antibiotic treatment and those who were given antibiotic treatment. Secondary bile acids were also significantly altered. At the same time, the differential microbial species/genera and the differential secondary bile acids interacted with each other.

Conclusions: This study identified several differential intestinal microbial species and secondary bile acids in fecal samples from infants with jaundice before and after phototherapy. Phototherapy can change the flora and its metabolism and its long-term impact needs further observation.

Key words: phototherapy, neonatal jaundice, intestinal flora, bile acids.

\section{Introduction}

Neonatal jaundice is a common condition worldwide, which may be physiological or pathological. Physiological jaundice results from hyperbilirubinemia, which presents as a high bilirubin concentration in the serum. Total serum bilirubin (TSB) is currently used as a diagnostic index for jaundice [1]. Most bilirubin comes from red blood cells (RBCS), which undergo hemolysis and release their hemoglobin content, with unconjugated bilirubin being the end-product of hemoglobin metabolism. After binding to serum albumin, unconjugated bilirubin is transported to the liver, where it will detach from the albumin and conjugate with glucuronic acid [2]. Conjugated bilirubin is water soluble and excreted into the small intestine with bile acids and then is further catabolized by colonic

\author{
Corresponding author: \\ Jinping Zhang \\ Department of Pediatrics \\ Shanghai Jiao Tong \\ University Affiliated \\ Sixth People's Hospital \\ 222 Huanhuxisan Road \\ Pudong New Area 201306 \\ Shanghai, China \\ Phone: +17621710577 \\ E-mail: zhang-jin-ping@163. \\ com
}


flora to urobilinogen, which is excreted from the body [3].

However, newborns have an increased bilirubin production because of the shorter lifespan of their RBCs and higher concentration of hemoglobin. On the other hand, newborns have a poorer capacity for the clearance of bilirubin. At birth, infants also have significantly lower levels of plasma albumin compared to adults [4]. The non-albumin-bound unconjugated bilirubin will pass through the blood-brain barrier and result in neuronal injury [5]. In the small intestine, the flora converts bilirubin monoglucuronides to urobilinogen, which is excreted from the body. In addition, the metabolites produced by intestinal microbiota contribute to the motility of the gastrointestinal tract (GIT). Nevertheless, newborns lack intestinal flora, and thus have a decreased intestinal motility [6]. Thus, a proportion of the bilirubin is deconjugated by $\beta$-glucuronidase and reabsorbed into the blood from the intestine [7].

Phototherapy is a treatment widely used to reduce serum bilirubin in jaundiced newborns, due to the safety and convenience of its use. As a yellow pigment, bilirubin strongly absorbs blue light in the $460 \mathrm{~nm}$ wavelength and then converts it irreversibly to lumirubin, a water-soluble and less toxic compound, which can be excreted with bile acids and urine [2].

The human GIT microbiota contributes to host metabolism. GIT microbiota produces short-chain fatty acids (SCFAs) and secondary bile acids, which play essential roles in host energy metabolism [8]. Primary bile acids, including cholic acid (CA) and chenodeoxycholic acid (CDCA), are synthesized in the liver and then converted to secondary bile acids by microbiota in the GIT [9-11]. Bile acids not only play roles in several metabolism processes, but also regulate the composition of the GIT microbiota [12]. In short, microbial richness is considered to be an indicator of a host's heath status. Alterations to the microbiota may lead to functional amelioration of the GIT microbiota's capacity to produce metabolites.

In the present study, the aim was to assess the benefit of treatment for hyperbilirubinemia with phototherapy. In this context, we characterized the $16 \mathrm{~S}$ rRNA microbiome of jaundiced infants before and after phototherapy, with or without antibiotic treatment. We also quantified the concentrations of secondary bile acids and determined the relationship between the differential microbiota species and differential secondary bile acids. We observed several species/ genera and secondary bile acids which may well participate in the clearance of bilirubin in jaundiced infants after they had been treated with phototherapy.

\section{Material and methods}

\section{Study cohort and patient characteristics}

Fifty-three term neonates with jaundice were treated in the neonatal intensive care unit of the Pediatrics Department, Hospital between June 2017 and June 2018. The whole cohort comprised infants with birth weight $\geq 2,500 \mathrm{~g}$ and gestational age over 37 weeks. This study was approved by the ethics committee of our hospital. Written consent was obtained from the legal guardians of these neonates before they were included in the study. All neonates with jaundice were diagnosed according to the criteria defined in practical neonatology. Briefly, neonates with the following symptoms were diagnosed with jaundice. (1) jaundice appears within $24 \mathrm{~h}$ after birth; (2) a serum bilirubin concentration > $221 \mu \mathrm{mol} / \mathrm{l}(12.9 \mathrm{mg} / \mathrm{dl})$, which was mainly indirect bilirubin, or the daily increase exceeded $85 \mathrm{\mu mol} / \mathrm{l}(5 \mathrm{mg} / \mathrm{dl})$; (3) jaundice appears again after receding; (4) jaundice lasted for $>2$ weeks and the serum conjugated bilirubin concentration was $>34 \mu \mathrm{mol} / \mathrm{l}(2 \mathrm{mg} / \mathrm{dl})$. Exclusion criteria were: congenital malformation; surgical disease; chromosomal abnormalities; congenital metabolic abnormalities; neonatal asphyxia; sepsis or suspected sepsis; $\mathrm{RH}$ hemolysis requiring blood exchange; glucose-6-phosphate dehydrogenase (G6PD) deficiency; hemoglobin disease or congenital infection.

\section{Treatment}

The blue LED light of the jaundice treatment apparatus with a double-sided light source (peak wavelength $425-475 \mathrm{~nm}$ ) was used. The blue light source was about $40 \mathrm{~cm}$ away from the neonates. During phototherapy for $24 \mathrm{~h}$ or $48 \mathrm{~h}$ the whole body, except for the genitals and eyes, was fully exposed to the light. Breathing and heart rate were closely monitored during the phototherapy treatment.

\section{Determination of serum bilirubin concentration}

The routine biochemistry panel, including serum concentrations of bilirubin, was carried out. The fifth-generation Total Bile Acids Randox RX Series assay (Randox Laboratories, Crumlin, UK) was used to measure the total concentrations of bile acids in sera.

\section{Fecal sample collection and storage}

In this pilot study, only a subset of 8 samples at baseline, 9 samples after $24 \mathrm{~h}$ phototherapy and 12 samples after phototherapy for $48 \mathrm{~h}$ were selected for analysis. Samples were selected randomly. Sterile containers were used to collect the fecal 
samples. Samples were collected on the day of hospital admission, and $48 \mathrm{~h}$ and $72 \mathrm{~h}$ after phototherapy. Samples were immediately frozen at $-20^{\circ} \mathrm{C}$ and stored until required for subsequent analysis.

\section{Extraction of fecal genomic DNA}

Fecal samples were collected in sterile containers and stored at $-20^{\circ} \mathrm{C}$ until required for further analysis. Genomic DNA was purified using a QIAamp PowerFecal DNA Kit according to the instructions of the manufacturer. The libraries were constructed using a standard protocol and the sequencing was produced on the Illumina Hiseq system. Sequence reads were assembled with SOAP de novo. The relative abundance of the microbial species was analyzed using MetaPhIAn.

\section{Quantification of fecal bile acids}

The major bile acids (BAs) were identified using gas chromatography. For protein precipitation, each $100 \mathrm{mg}$ fecal sample was mixed with $0.3 \mathrm{ml}$ of methanol, followed by vortex mixing for $1 \mathrm{~min}$. After 10 min centrifugation $(12,000 \mathrm{~g})$ at $4^{\circ} \mathrm{C}$, the supernatant was transferred and diluted 10 times in methanol, followed by another $1 \mathrm{~min}$ of vortex. After a further additional 10 min centrifugation $(12,000 \mathrm{~g})$ at $4^{\circ} \mathrm{C}$, the supernatant $(5 \mu \mathrm{l})$ was injected into the UPLC (Waters ACQUITY UPLC, US) for analysis.

Chromatographic separation was accomplished in a Thermo Ultimate 3000 system equipped with an ACQUITY UPLC BEH C18 $(100 \times 2.1 \mathrm{~mm}, 1.7 \mu \mathrm{m})$ Waters column maintained at $40^{\circ} \mathrm{C}$. Gradient elution of analytes was carried out with $0.01 \%$ formic acid in water (A) and acetonitrile (B). An increasing linear gradient of solvent $B(v / v)$ was used as follows: 0-4 $\min , 25 \% \mathrm{~B}$; 4-9 $\mathrm{min}, 25-30 \% \mathrm{~B}$; 9-14 min, 30-36\% B; $14-18 \mathrm{~min}, 36-38 \% \mathrm{~B}$; 18-24 min, 38-50\% B; 24-32 min, $50-75 \% \mathrm{~B}$; 32-35 min, $75-100 \%$ B; 35-38 min, $100-25 \%$ B. The capillary temperature was $500^{\circ} \mathrm{C}$. The Orbitrap analyzer scanned over a mass range of $\mathrm{m} / \mathrm{z} 81-$ 1000 for a full scan at a mass resolution of 70,000.

Data dependent acquisition (DDA) MS/MS experiments were performed with an HCD scan. The normalized collision energy was $30 \mathrm{eV}$. Dynamic exclusion was implemented to remove some unnecessary information in the MS/MS spectra.

\section{Ethics approval}

The study was approved by the Ethics Committee of Shanghai Sixth People's Hospital East Campus. Approval No: 2017-009.

\section{Statistical analysis}

SPSS 23 was used to analyze the data, which are presented as the mean \pm SE. The Wilcoxon rank sum test was employed for comparisons between groups with a non-normal distribution. Correlations between variables were evaluated using the Spearman coefficient. A $p$-value $<0.05$ was considered to be a statistically significant finding.

\section{Results}

\section{Clinical characteristics of infants with jaundice}

Of the 53 jaundiced infants enrolled in the study, all had been admitted to the NICU of the Sixth People's Hospital Affiliated to Jiaotong University, Shanghai, between June 2017 and June 2018. The average gestational age was $271.2 \pm 8.77$ days and birth weight $3.2 \pm 0.36 \mathrm{~kg}$. The onset of jaundice occurred $2.17 \pm 1.43$ days after birth. The mean total serum bilirubin (TSA) was $19.7 \pm 3.7 \mathrm{mg} / \mathrm{dl}$, and transcutaneous bilirubin (TCB) concentration was $21.49 \pm 4.36$ $\mathrm{mg} / \mathrm{dl}$. Twenty-nine of the babies were treated with antibiotics, and the others were not (Table I).

\section{Phototherapy effects on total serum bilirubin}

The concentration of total serum bilirubin was measured at baseline and after treatment. At the time of hospitalization, the mean serum bilirubin of all the patients combined was $19.7 \pm 3.7 \mathrm{mg} / \mathrm{dl}$. It was $19.2 \pm 2.8 \mathrm{mg} / \mathrm{dl}$ and $20.1 \pm 4.3 \mathrm{mg} / \mathrm{dl}$ in the non-antibiotic-treated group and antibioticstreated group, respectively. As shown in Table II, the phototherapy cleared the bilirubin significantly. In the group of non-antibiotic-treated patients, the mean serum bilirubin level decreased to 13.7 $\pm 2.7 \mathrm{mg} / \mathrm{dl}$ and $0.8 \pm 0.2 \mathrm{mg} / \mathrm{dl} 24 \mathrm{~h}$ and $48 \mathrm{~h}$ after phototherapy treatment, respectively. In the group of antibiotic-treated infants, it decreased to 13.2 $\pm 3.2 \mathrm{mg} / \mathrm{dl}$ and $0.7 \pm 0.2 \mathrm{mg} / \mathrm{dl} 24 \mathrm{~h}$ and $48 \mathrm{~h}$ after

Table I. Baseline and clinical characteristics $(n=40)$

\begin{tabular}{|c|c|}
\hline Parameter & Value \\
\hline \multicolumn{2}{|c|}{ Birth characteristics, mean \pm SD: } \\
\hline Gestational age [days] & $271.20 \pm 8.77$ \\
\hline Birth weight $[\mathrm{kg}]$ & $3.2 \pm 0.36$ \\
\hline Onset of jaundice [days] & $2.18 \pm 1.40$ \\
\hline Age [days] & $7.10 \pm 5.24$ \\
\hline Phototherapy duration [h] & $88.51 \pm 41.92$ \\
\hline $\mathrm{TCB}[\mathrm{mg} / \mathrm{dl}]$ & $21.49 \pm 4.36$ \\
\hline $\mathrm{TSA}[\mathrm{mg} / \mathrm{dl}]$ & $19.7 \pm 3.7$ \\
\hline \multicolumn{2}{|l|}{ Feeding type, $n(\%)$ : } \\
\hline Breastfeeding & $36(67.92)$ \\
\hline Mixture & $17(32.08)$ \\
\hline \multicolumn{2}{|l|}{ Antibiotics usage, $n(\%)$ : } \\
\hline Yes & $29(54.72)$ \\
\hline No & $24(45.28)$ \\
\hline
\end{tabular}


Table II. Effects of phototherapy on total serum bilirubin concentrations before and after phototherapy

\begin{tabular}{|lccc|}
\hline Antibiotics usage & Baseline $[\mathrm{mg} / \mathrm{dl}]$ & Phototherapy $24 \mathrm{~h}[\mathrm{mg} / \mathrm{dl}]$ & Phototherapy $\mathbf{4 8} \mathbf{~ h ~} \mathbf{m g} / \mathbf{d l}]$ \\
\hline No $(24$ cases $)$ & $19.2 \pm 2.8(24$ cases $)$ & $13.7 \pm 2.7(24$ cases $)$ & $0.8 \pm 0.2(21$ cases $)$ \\
\hline Yes $(29$ cases $)$ & $20.1 \pm 4.3(29$ cases $)$ & $13.2 \pm 3.2(29$ cases $)$ & $0.7 \pm 0.2(20$ cases $)$ \\
\hline
\end{tabular}
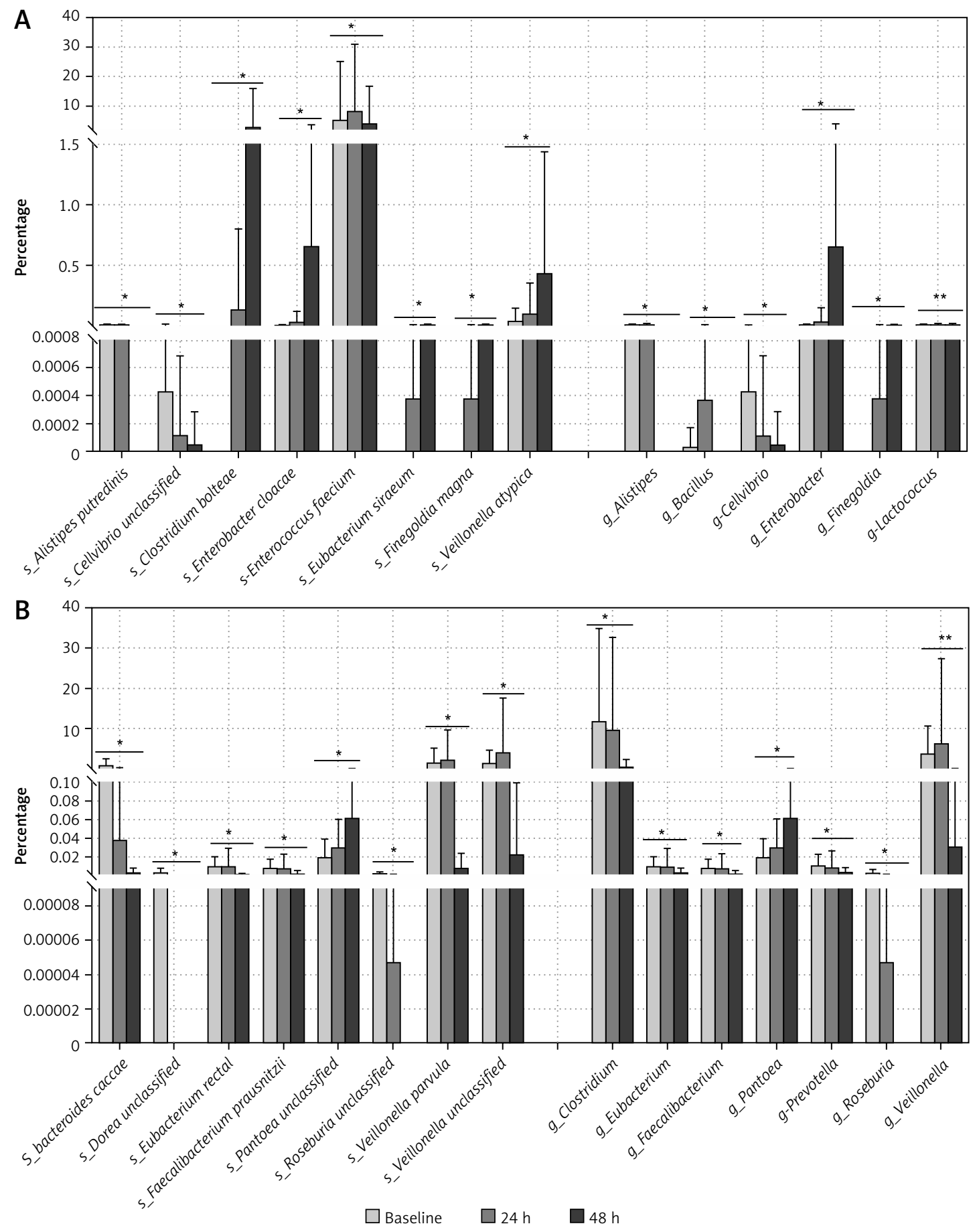

Figure 1. Differential gut microbial species and genera before and after phototherapy, as characterized by 16S rRNA sequencing. A - Samples are from infants without antibiotic treatment. B - Samples are from infants with antibiotic treatment 
phototherapy, respectively. Furthermore, there was no significant difference between the two groups.

\section{Phototherapy effects on abundance of microbiota in the GIT}

To evaluate the alteration of GIT microbiota before and after phototherapy, Macrogene sequencing analysis was employed. Among the infants who did not receive antibiotic treatment (Figure $1 \mathrm{~A}$ ), many species detected were significantly altered. Alistipes putredinis and Cellvibrio unclassified were found to be decreased after phototherapy, while Clostridium bolteae, Enterobacter cloacae, Enterococcus faecium, Eubacterium siraeum, Finegoldia magna and Veillonella atypica were found to be increased significantly. Genera including Alistipes, Bacillus, Cellvibrio, Enterobacter, Finegoldia and Lactococcus were altered significantly after phototherapy. On the other hand, among the infants who were given antibiotic treatment (Figure $1 \mathrm{~B}$ ), species were also found to have been altered significantly after phototherapy. Many species increased including Bacteroides caccae, Dorea unclassified, Eubacterium rectale, Faecalibacterium prausnitzii, Roseburia unclassified, Veillonella parvula, and Veillonella unclassified. Only Pantoea unclassified was found to be decreased after phototherapy. The genera included Clostridium, Eubacterium, Faecalibacterium, Pantoea, Prevotella, Roseburia and Veillonella. These results indicated that phototherapy could modulate the relative abundance of some species and genera. It is noteworthy that the differential species or genera were quite different in infants who were treated with antibiotics and those who were not.

\section{Phototherapy effects on relative abundance} of BAs

The microbiota metabolizes the primary bile acids to secondary BAs. LC/MS was used to evaluate the fecal BA profiles in the infants enrolled in the study. Figure 2 demonstrates that phototherapy could induce alterations in some types of secondary bile acids, as indicated by analysis of the fecal samples. In the group of non-antibiotic-treated infants (Figure 2), the concentrations of 11 secondary bile acids were significantly altered after phototherapy, including LCA, 12-ketol LCA, $\beta$-UDCA, DCA, NorCA, GLCA, GUDCA, GDCA, TLCA, THDCA and TUDCA. All differential secondary bile acids were elevated $24 \mathrm{~h}$ after phototherapy and then reduced to a lower level $48 \mathrm{~h}$ after phototherapy, as compared with baseline. On the other hand, in the group of antibiotic-treated infants (Figure 3), levels of 8 secondary bile acids were altered after phototherapy, i.e. LCA, isoLCA, NorCA, GLCA, GUDCA, GDCA, LCA-3S and TLCA. In detail, levels of LCA and LCA-3S were reduced $24 \mathrm{~h}$ or $48 \mathrm{~h}$ after phototherapy compared to baseline. isoLCA, NorCA, GLCA, GUDCA, GDCA and TLCA were elevated $24 \mathrm{~h}$ after phototherapy and then were reduced to lower concentrations $48 \mathrm{~h}$ after phototherapy compared to baseline values. These results strongly suggested that phototherapy could affect the production of bile acids.

\section{Impact of differential species or genera on differential bile acids}

To explore further the association between the differential species/genera and the differential secondary bile acids, multivariate logistic regression analyses were conducted. First, we assessed the effect of differential species or genera on bile acids' alteration when adjusting for the use of probiotics or breastfeeding. Notably, among the infants who did not receive antibiotic treatment (Figure $4 \mathrm{~A}$ ), the alteration of the genus Bacillus was associated with $\beta$-UDCA (OR: $1.2115 \times 10^{17}$, $\left.95 \% \mathrm{Cl}: 1.5561 \times 10^{9}-9.4322 \times 10^{24}\right)$. Among the infants given antibiotics (Figure $4 \mathrm{~B}$ ), the alteration of LCA was associated with Bacteroides caccae $(\mathrm{OR}=0.0033,95 \% \mathrm{Cl}: 0.0003-0.0342)$, Veillonella parvula $(\mathrm{OR}=66122791.02,95 \% \mathrm{Cl}$ : 134159.7-32589693406), Veillonella unclassified $\left(\mathrm{OR}=2.4282 \times 10^{-8}, 95 \% \mathrm{Cl}: 5.3115 \times 10^{-}\right.$ ${ }^{11}-1.1100 \times 10^{-5}$ ) and the genus Veillonella (OR $\left.=0,95 \% \mathrm{Cl}: 0-6.3239 \times 10^{-271}\right)$. The alteration of LCA-3S was associated with Bacteroides caccae $\left(\mathrm{OR}=1.6320 \times 10^{-5}, 95 \% \mathrm{Cl}: 2.9602 \times 10^{-6}-8.9978\right.$ $\left.\times 10^{-5}\right)$, Roseburia unclassified $(\mathrm{OR}=0,95 \% \mathrm{Cl}$ : 0-0), Veillonella parvula (OR $=145,925.7967$, 95\% Cl: 1,569.550155-13,567,160.04), Veillonella unclassified $(\mathrm{OR}=0.000547188,95 \% \mathrm{Cl}: 6.2182$ $\left.\times 10^{-6}-0.04815\right)$. The alteration of GDCA was associated with Pantoea unclassified (OR $=1.3752$, 95\% Cl: 1.2344-1.5320).

\section{Impact of differential bile acids on differential species or genera}

Next, we assessed the effect of differential bile acids on differential species or genera after adjusting the data for the use of probiotics or breast-feeding. Notably, among the infants who were not treated with antibiotics (Figure $5 \mathrm{~A}$ ), the alteration of the species Eubacterium siraeum was associated with 12-ketoLCA (OR $=1.0233$, 95\% Cl: 1.0017-1.0453), DCA (OR $=1.0074$, 95\% Cl: $1.0012-1.0136)$ and GLCA (OR $=0.7460$, 95\% Cl: $0.5785-0.9619, p<0.05)$. The alteration of the genus Bacillus was associated with $\beta$-UDCA $(\mathrm{OR}=1.0088,95 \% \mathrm{Cl}: 1.0030-1.0146)$, DCA $(\mathrm{OR}=1.0043,95 \% \mathrm{Cl}: 1.0015-1.0071)$ and GDCA $(\mathrm{OR}=0.917,95 \% \mathrm{Cl}: 0.8680-0.9692)$. On the other hand, alteration of the genus Lactococcus was 

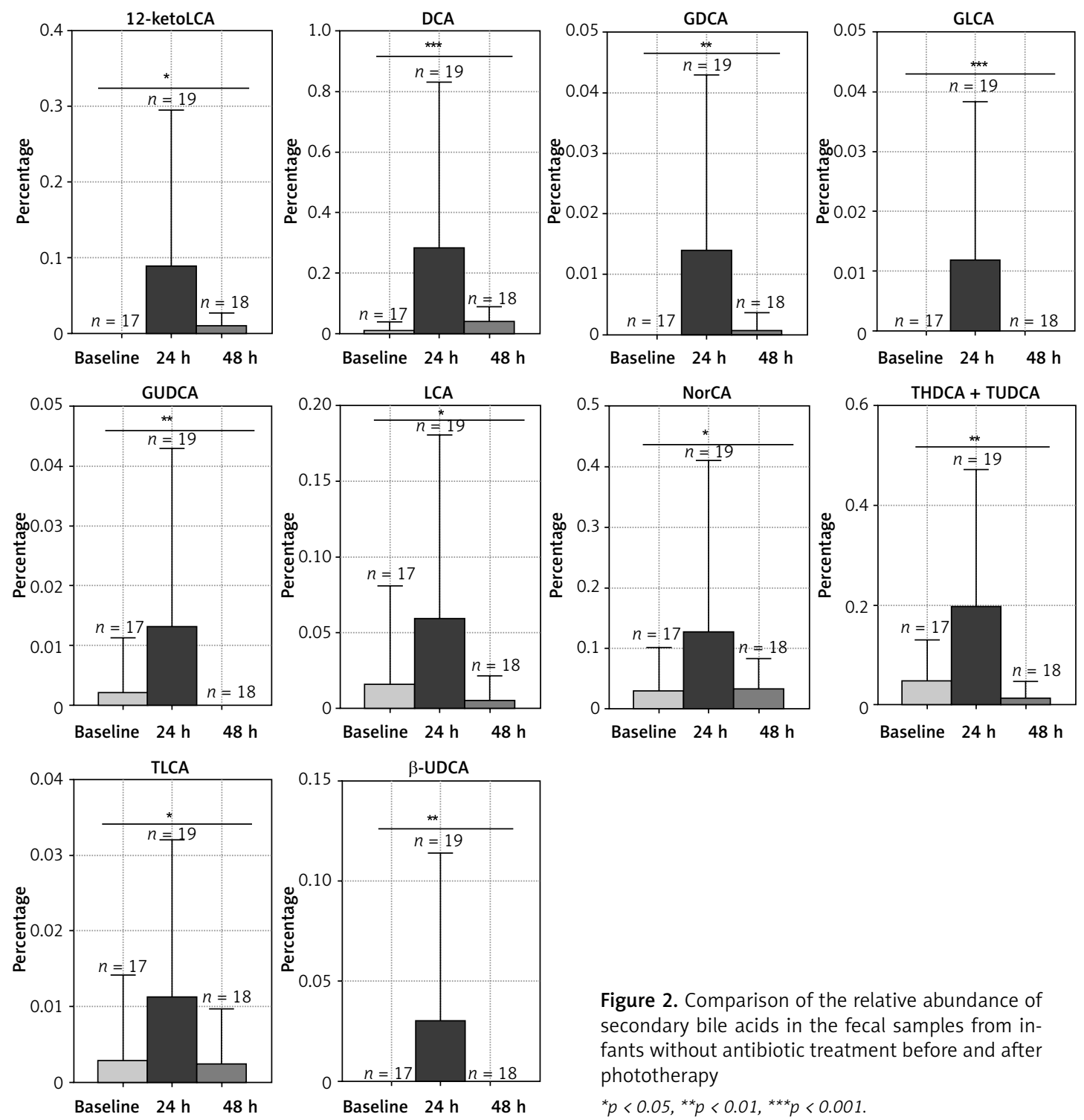

associated with GLCA alteration $(O R=0.1440$ 95\% Cl: 0.0318-0.6512). In contrast, among the infants given antibiotics (Figure $5 \mathrm{~B}$ ), the species Bacteroides caccae was associated with isoLCA $\left(\mathrm{OR}=3.2794 \times 10^{16}, 95 \% \mathrm{Cl}: 5.4541 \times 10^{13}\right.$ $\left.1.9718 \times 10^{19}\right)$ and GLCA $\left(\mathrm{OR}=2.6089 \times 10^{-64}\right.$, $\left.95 \% \mathrm{Cl}: 2.6234 \times 10^{109}-2.5944 \times 10^{-19}\right)$. Also, the species Eubacterium rectale $(\mathrm{OR}=1.6634$, 95\% Cl: 1.2759-2.1685) and Faecalibacterium prausnitzii $(\mathrm{OR}=1.5533,95 \% \mathrm{Cl}: 1.2476-1.9340)$ and the genera Eubacterium $(\mathrm{OR}=1.6642$, 95\% Cl: 1.2755-2.1712), Faecalibacterium (OR = $1.5533,95 \% \mathrm{Cl}: 1.2476-1.9340$ ), and Prevotella $(\mathrm{OR}=1.6330,95 \% \mathrm{Cl}: 1.2486-2.1357)$ were associated with alterations in TLCA.

\section{Discussion}

The neonatal intestinal microbiota was influenced by several factors including diet, the maternal microbiome and local culture, which may

Figure 2. Comparison of the relative abundance of secondary bile acids in the fecal samples from infants without antibiotic treatment before and after phototherapy ${ }^{*} p<0.05,{ }^{* *} p<0.01,{ }^{* *} p<0.001$.

have produced geographical differences in the microbiota composition in the cohort of infants [13]. However, neonatal jaundice is a common clinical sign in newborn infants worldwide, for which phototherapy is the simplest treatment. Hence, it will be necessary to determine the GIT microbiota composition in jaundiced infants before and after phototherapy in different regional-based cohorts. Antibiotics are widely used in neonatal medication, but they perturb a developing infant's intestinal microbiota. In the present study, infants treated with antibiotics or not had quite a different differential species/genera before and after phototherapy, which supports the disruption effect of antibiotic therapy. More importantly, various factors, including antibiotic-resistant genes, should be taken into consideration when trying to understand the responses of infants to antibiotics.

Phototherapy could transform the unconjugated bilirubin into conjugated forms, which could 

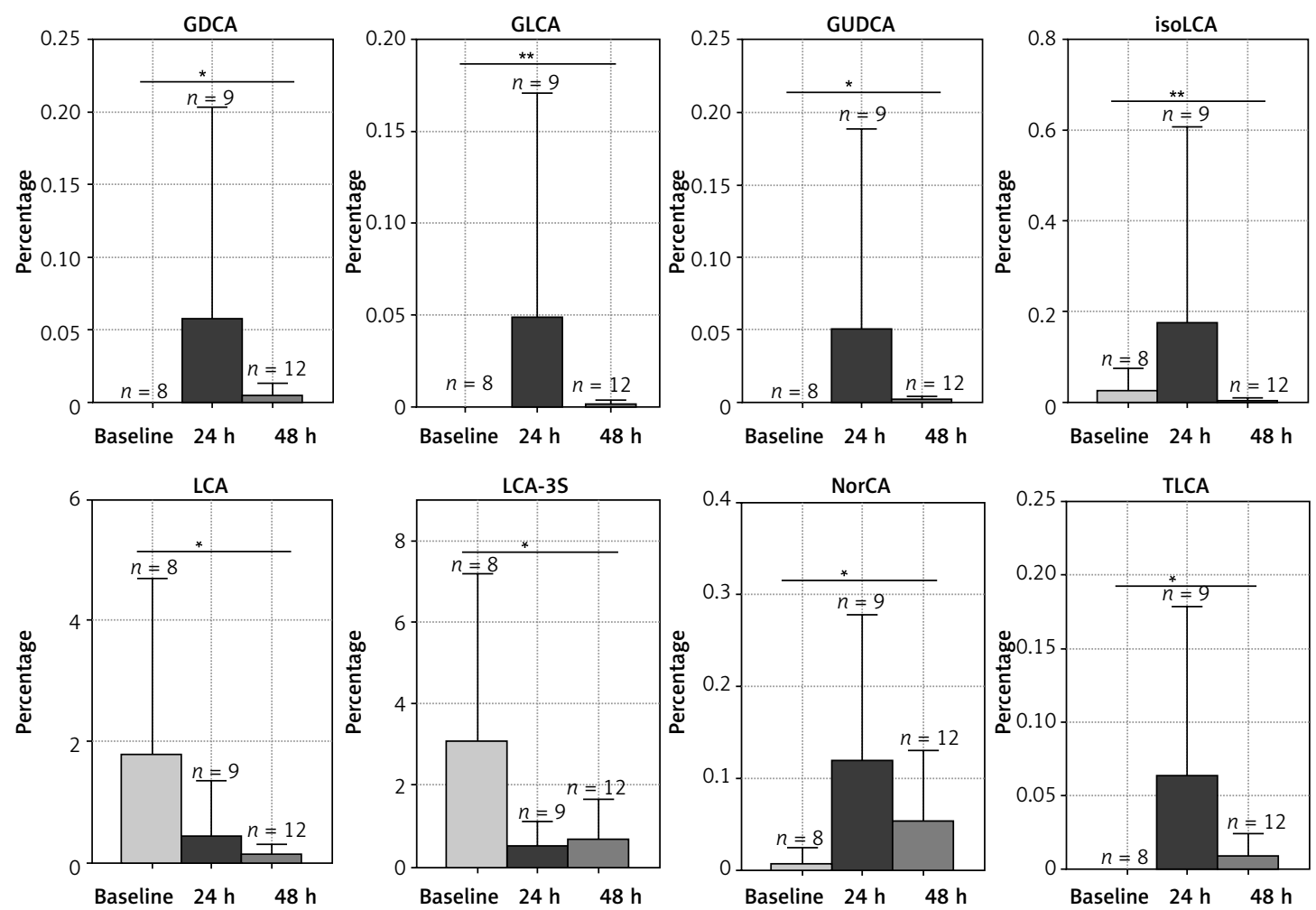

Figure 3. Comparison of the relative abundance of secondary bile acids in the fecal samples from infants with antibiotic treatment before and after phototherapy ${ }^{*} p<0.05,{ }^{* *} p<0.01,{ }^{* * *} p<0.001$.

then be metabolized to urobilinoids by the GIT microbiota. In our study, the TSB of the jaundiced infants dropped drastically in the first $24 \mathrm{~h}$ after phototherapy, and then returned to normal values $48 \mathrm{~h}$ after phototherapy. By contrast, the relative abundance of fecal secondary bile acids rose rapidly in the first $24 \mathrm{~h}$ after phototherapy, but decreased to the level of baseline in the second $24 \mathrm{~h}$. Accordingly, the correlated microbiota species also exhibited a higher relative abundance $24 \mathrm{~h}$ after phototherapy compared to baseline and then a reduction $48 \mathrm{~h}$ after phototherapy. This finding suggested that TSB reduction in the first $24 \mathrm{~h}$ is sufficient to induce the alteration of differential species/genera and secondary bile acids. The underlying mechanisms of this response remain to be investigated.

Reduction of enterohepatic circulation by accelerated bilirubin fecal excretion can reduce infant jaundice. It is well known that exposure to phototherapy provokes an increased rate of intestinal transit and thus diarrhea in jaundiced infants [14, 15]. It is worth thinking about how phototherapy can induce an alteration in the GIT microbiota species. Previous studies have indicated a significantly greater colon motility index for patients with diarrhea, as excess bile acid concentrations in the colon stimulate enteroendocrine cells and accelerate colonic transit. In our study, we observed elevation of fecal secondary bile acids after photo- therapy, which leads to speculation that bilirubin metabolism after phototherapy might modulate colonic microbiota species and thus produce more fecal secondary bile acids accordingly. There is limited information on the mechanisms involved in the colon motility response to secondary bile acids. However, the bile acid-GIT microbiome axis has emerged as an important regulator of health and findings have indicated that species interacted with each other. Indeed, we find relationships between differential secondary bile acids and differential species/genera. TLCA (taurolithocholic acid) and DCA are activators of Gas-protein-coupled receptor $G$ protein-coupled bile acid receptor1 (TGR5), whose activation protects the intestinal barrier function and reduces inflammation [16]. We found that changes in TLCA composition had an impact on several differential species alterations after phototherapy. Among them, Eubacterium rectale, Faecalibacterium prausnitzii, and the genus Prevotella, which are SCFA-producing bacteria [17, 18], were affected by TLCA. Several studies have reported that fecal DCA could be increased by a western diet and participate in intestinal carcinogenesis [19]. Nevertheless, carcinogenesis is a long-term process with stepwise accumulation of mutations. Therefore, transient elevation of DCA after phototherapy might be a stress reaction, and how it modulates GIT homeostasis after phototherapy remains to be explored. 
A

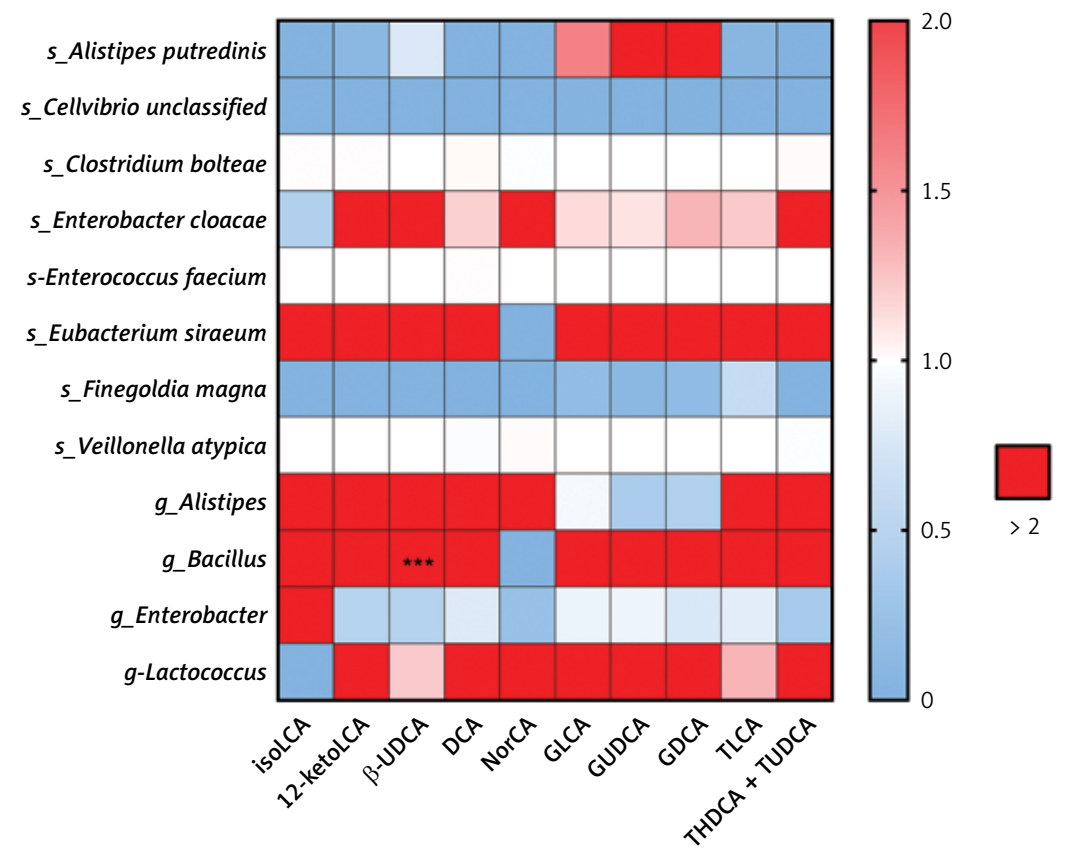

B

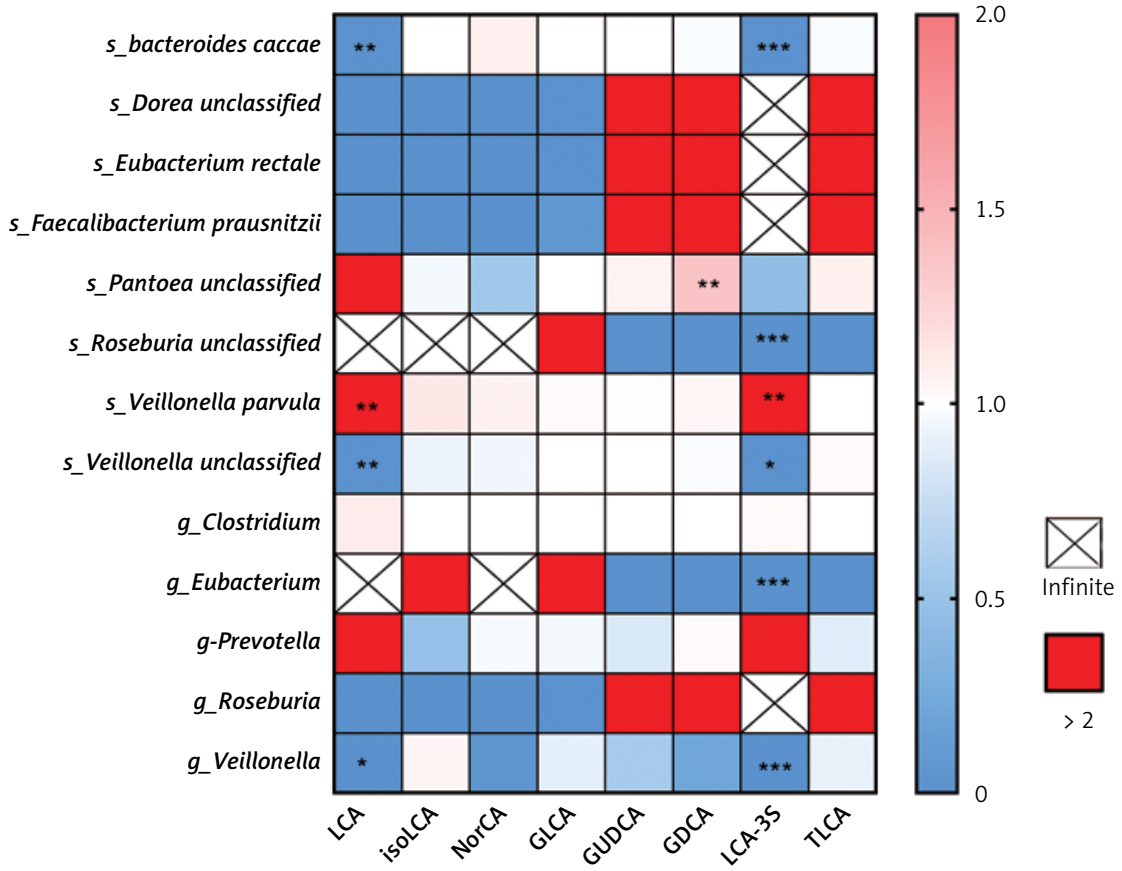

Figure 4. Differential gut microbial species/genera impact on secondary bile acids. A - Heatmap of odds ratio between differential gut microbial and differential secondary bile acids in infants without antibiotic treatment. B - Heatmap of odds ratio between differential gut microbial and differential secondary bile acids in infants with antibiotic treatment. Rows represent differential microbial species/genera and columns represent differential secondary bile acids

${ }^{*} p<0.05,{ }^{\star *} p<0.01,{ }^{* \star *} p<0.001$.

In conclusion, the present study revealed fluxes in bile acids and their association with differential species/genera before and after phototherapy treatment. We believe that a large-cohort study needs to be conducted to confirm the physiological roles of these differential species and BAs.

\section{Acknowledgments}

Kun Zhang and Sainan Fan contributed equally to this work.

This study was supported by the people's livelihood research special funding of Shanghai Science and technology development (PKJ2017-Y09). This study was supported by Clinical Research 
A

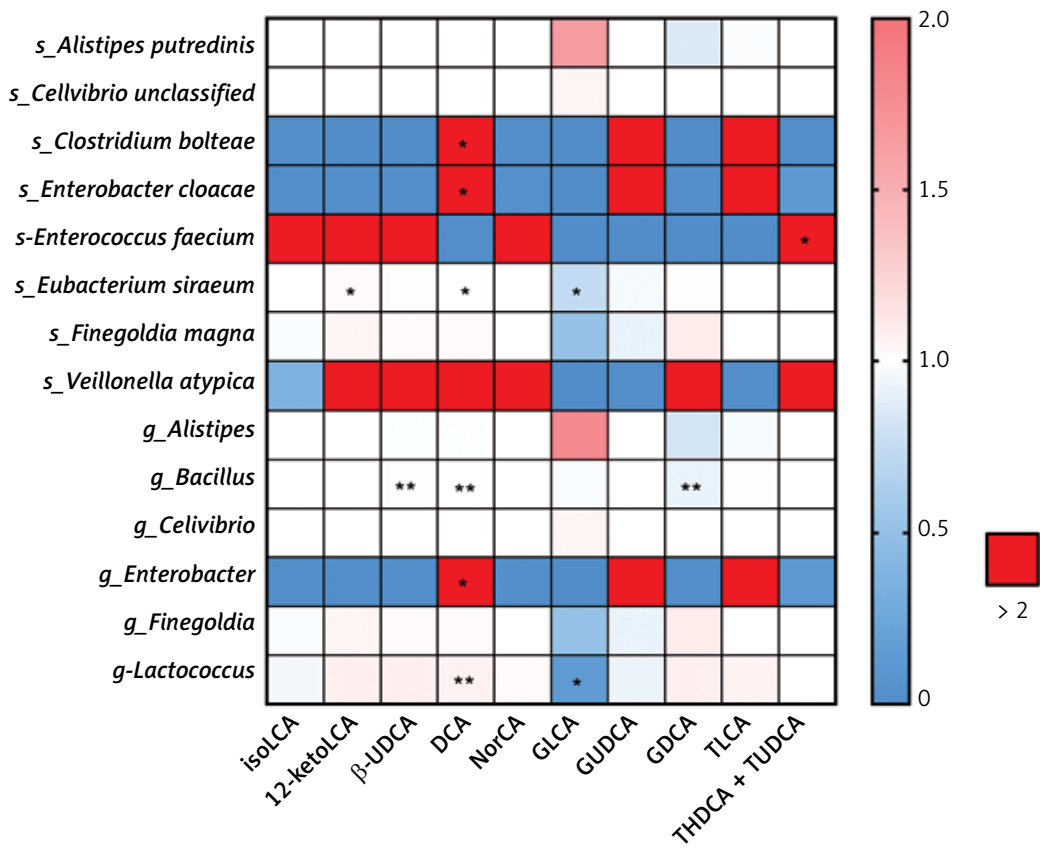

B

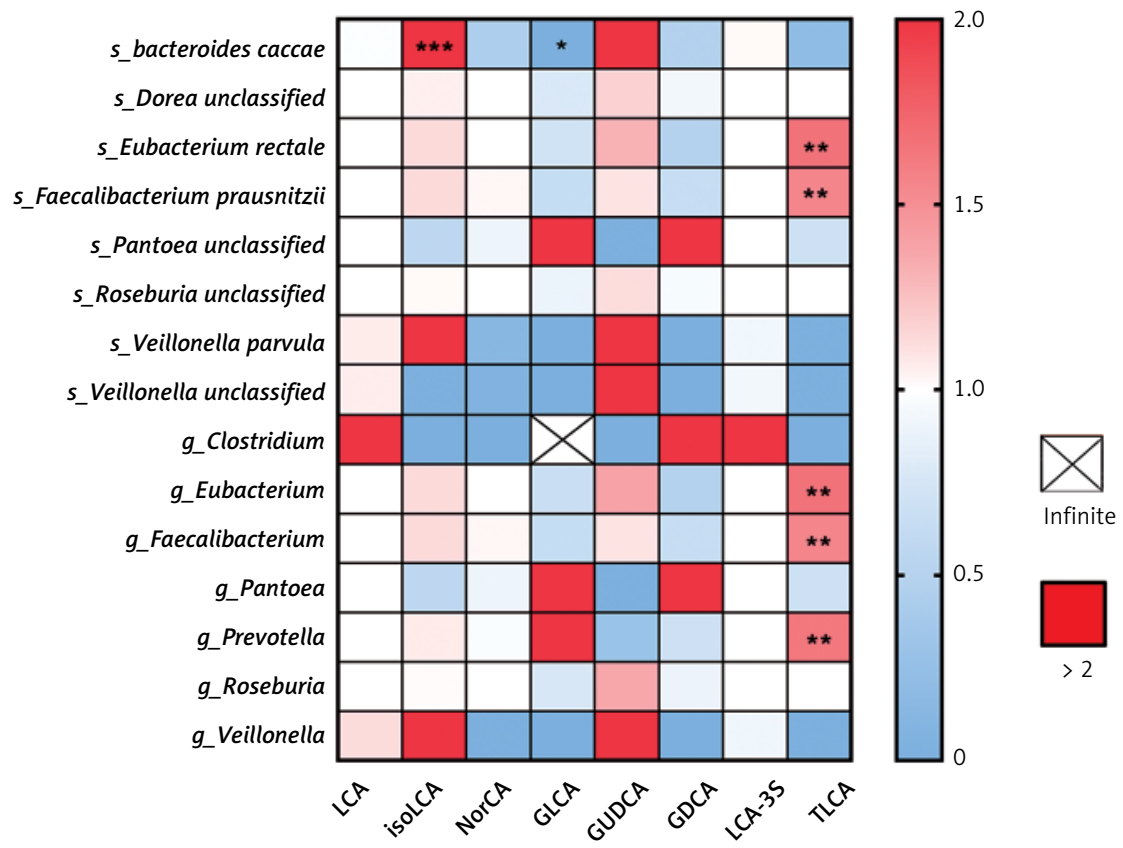

Figure 5. Differential secondary bile acids' impact on gut microbial species/genera. A - Heatmap of odds ratio between differential gut microbial and differential secondary bile acids in infants without antibiotic treatment. B - Heatmap of odds ratio between differential gut microbial and differential secondary bile acids in infants with antibiotic treatment. Rows represent differential microbial species/genera and columns represent differential secondary bile acids

${ }^{*} p<0.05,{ }^{* *} p<0.01,{ }^{* *} p<0.001$.

Plan of SHDC (SHDC2020CR3055B). This study was supported by Shanghai Pudong Health and Family Planning Commission Joint Public Relations Project (PW2017D-7). The funding organization played no role in the implementation of this study.

\section{Conflict of interest}

The authors declare no conflict of interest.

\section{References}

1. Watson RL. Hyperbilirubinemia. Crit Care Nurs Clin North Am 2009; 21: 97-120. 
2. Dennery PA, Seidman DS, Stevenson DK. Neonatal hyperbilirubinemia. N Engl J Med 2001; 344: 581-90.

3. Takimoto M, Matsuda I. Glucruonidase activity in the stool of the newborn infant. Biol Neonate 1971; 18: 66-70.

4. Notarianni LJ. Plasma protein binding of drugs in pregnancy and in neonates. Clin Pharmacokinet 1990; 18: 20-36.

5. Sinclair CJ. A difference in mortality rate and incidence of kernicterus among premature infants allotted to two prophylactic antibacterial regimens, by William A. Silverman, et al, Pediatrics, 1956; 18: 614-624. Pediatrics 1998; 102: 225-7.

6. Koppen IJN, Benninga MA, Singendonk MMJ. Motility disorders in infants. Early Hum Dev 2017; 114: 1-6.

7. Mitra S, Rennie J. Neonatal jaundice: aetiology, diagnosis and treatment. Br J Hosp Med 2017; 78: 699-704.

8. Kapourchali FR, Cresci GAM. Early-life gut microbiome - the importance of maternal and infant factors in its establishment. Nutr Clin Pract 2020; 35: 386-405.

9. Lefebvre P, Cariou B, Lien F, Kuipers F, Staels B. Role of bile acids and bile acid receptors in metabolic regulation. Physiol Rev 2009; 89: 147-91.

10. Staels B, Fonseca VA. Bile acids and metabolic regulation: mechanisms and clinical responses to bile acid sequestration. Diabetes Care 2009; 32 Suppl 2: S237-45.

11. Kuver R. The expanding universe of bile acid physiology: delving into the mysteries of dark (green) matter. J Surg Res 2013; 182: 207-9.

12. Ridlon JM, Kang DG, Hylemon PB, Bajaj JS. Bile acids and the gut microbiome. Curr Opin Gastroenterol 2014; 30: 332-8.

13. Echarri PP, Graciá CM, Berruezo GR, et al. Assessment of intestinal microbiota of full-term breast-fed infants from two different geographical locations. Early Hum Dev 2011; 87: 511-3.

14. Sivan Y, Dinari G, Goodman C, Merlob P, Nitzan M. Small intestine transit time and lactose absorption during phototherapy. Biol Neonate 1985; 48: 10-4.

15. Rubaltelli F, Largajolli G. Effect of light exposure on gut transit time in jaundiced newborns. Acta Paediatr Scand 1973; 62: 146-8.

16. Brighton CA, Rievaj J, Kuhre RE, et al. Bile acids trigger GLP-1 release predominantly by accessing basolaterally located g protein-coupled bile acid receptors. Endocrinology 2015; 156: 3961-70.

17. Ferreira-Halder V, Faria AVS, Andrade SS. Action and function of Faecalibacterium prausnitzii in health and disease. Best Pract Res Clin Gastroenterol 2017; 31: 643-8.

18. Louis P, Flint HJ. Diversity, metabolism and microbial ecology of butyrate-producing bacteria from the human large intestine. FEMS Microbiol Lett 2009; 294: 1-8.

19. Jenkins GJ, Cronin J, Alhamdani A, et al. The bile acid deoxycholic acid has a non-linear dose response for DNA damage and possibly NF-kappaB activation in oesophageal cells, with a mechanism of action involving ROS. Mutagenesis 2008; 23: 399-405. 